\title{
Characterization of Nano-Oxide Layers Fabricated by Ion Beam Oxidation
}

\author{
Susana Cardoso, Zongzhi Zhang, Haohua Li, Ricardo Ferreira, Paulo P. Freitas, Member, IEEE, Peng Wei, \\ José C. Soares, Etienne Snoeck, and Xavier Batlle
}

\begin{abstract}
In this paper, a remote $\mathrm{O}_{2}$ ion source is used for the formation of nano-oxide layers. The oxidation efficiency was measured in $\mathrm{CoFe}$-oxide films, and a decrease of the oxide layer with the pan angle and the oxidation pressure is observed. For the same oxidation pressure, the oxidation efficiency depends on the $\mathrm{O}_{2}$ content in the $\mathrm{Ar}-\mathrm{O}_{2}$ plasma. These results were applied in optimizing the fabrication of $\mathrm{Al}_{2} \mathrm{O}_{3}$ barrier for tunnel junctions. This method was also used to fabricate junctions with $\mathrm{Fe}$-oxide layers inserted at the $\mathrm{Al}_{2} \mathrm{O}_{3}-\mathrm{CoFe}$ interface. TEM and magnetization data indicate that after anneal at $385^{\circ} \mathrm{C}$, a homogeneous ferromagnetic $\mathrm{Fe}$-oxide layer $\left(\mathrm{Fe}_{3} \mathrm{O}_{4}\right.$ ?) is formed.
\end{abstract}

Index Terms-Ion oxidation, nano-oxide layers, transmission electron microscopy, tunnel junctions.

\section{INTRODUCTION}

$\mathbf{T}$ HE fabrication of thin oxide layers $(<20 \AA)$ is commonly done by oxidation of a metallic layer, using natural or plasma oxidation [1]. The control of the oxidation process and the smoothness of the metallic layer will determine the continuity and quality of the oxide layer. In particular, for a magnetic tunnel junction application, the oxide barrier and the interface with the electrodes are determinant for the device operation. Also, the use of spin valves at recording densities $>100 \mathrm{Gbit} / \mathrm{in}^{2}$ requires magnetoresistive signals that approach $\sim 20 \%$, which can be obtained using specular spin valves, where nano-oxide layers are incorporated in the present state-of-the-art spin valves. The consequent increase of the sensor resistance becomes an advantage if a current-perpendicular-to-plane geometry is used.

The method for ion beam oxidation using remote plasma is described in this paper. Although the method has been used for tunnel junction fabrication for several years [2], this paper is focused on the characterization of the oxide layers formed by this

Manuscript received February 13, 2002; revised May 22, 2002. This work was supported by projects PRAXIS XXI/P/CTM/10220/98 and Sapiens 34116/99 and by SAIT under the Korean National Program for Tera-level Nano-devices.

S. Cardoso, Z. Zhang, H. Li, R. Ferreira, and P. P. Freitas are with the Instituto de Engenharia de Sistemas e Computadores, Microsystems and Nanotechnologies, Lisbon and also with Instituto Superior Técnico, Lisbon, Portugal (e-mail: susana.freitas@inesc.pt).

P. Wei was with the Instituto Tecnológico e Nuclear, Sacavém, Portugal $\mathrm{He}$ is now with the Advanced Science Research Center, Japan (e-mail: pengwei@taka.jaeri.go.jp).

J. C. Soares is with the Instituto Tecnológico e Nuclear, Sacavém and also with the Centro de Fisica Nuclear da Universidade de Lisboa, Portugal (e-mail: soaresjc@alf1.cii.fc.ul.pt).

E. Snoeck is with the CEMES-CNRS, Toulouse, France (e-mail: snoeck@cemes.fr).

$\mathrm{X}$. Batlle is with the Departemento Fisica Fondamental, Universitat Barcelona, Spain (e-mail: xavier@ffn.ub.es).

Digital Object Identifier 10.1109/TMAG.2002.802863.

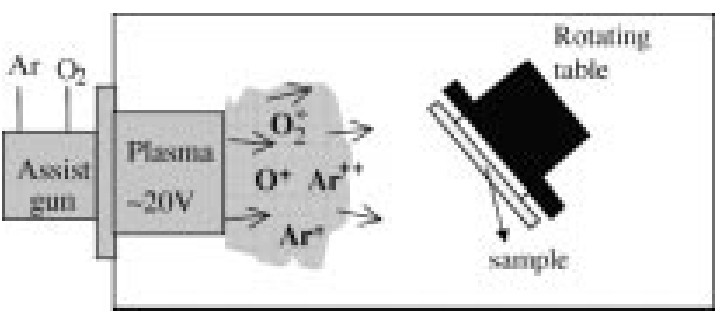

Fig. 1. Oxidation using a remote plasma created inside the assist source. Ar is required for plasma stabilization. The ions reach the sample surface without any voltage applied to the assist gun grids.

method. A particular application is shown for tunnel junctions fabricated with two nano-oxide layers: the $\mathrm{Al}_{2} \mathrm{O}_{3}$ barrier and a Fe-oxide layer inserted between the $\mathrm{Al}_{2} \mathrm{O}_{3}$ barrier and the top electrode [3]. With this FeOx layer, 40\% tunnel magnetoresistance (TMR) is obtained upon anneal at $380{ }^{\circ} \mathrm{C}$. This structure maintains TMR values of $20 \%$ after 10 -h anneal at $380{ }^{\circ} \mathrm{C}$.

\section{EXPERIMENT}

The structures studied were deposited using an automated Nordiko N3000 ion beam system [2] with a base pressure of $5 \times 10^{-8}$ torr. The depositions were done at $3.5 \times 10^{-5}$ torr, using an Xe beam (33 mA) formed by extracting the ions with $1750 \mathrm{~V}$ (acceleration $=+1450 \mathrm{~V}$, deceleration $=-300 \mathrm{~V}$ ) from an RF plasma created inside a $10-\mathrm{cm}$ diameter ion source. The oxidation (Fig. 1) was carried out by the ions from an $\mathrm{Ar}-\mathrm{O}_{2}$ plasma $\left(2.24 \mathrm{~kW} / \mathrm{m}^{2}\right)$ created inside the $25-\mathrm{cm}$ diameter assist source (Ar is needed for plasma stabilization). The pressure was varied from $3.2 \times 10^{-5}$ to $2.4 \times 10^{-4}$ torr and was determined by the flow of $\mathrm{Ar}$ and $\mathrm{O}_{2}$, which was tuned from 4-16 sccm (Ar) and $1.5-40 \mathrm{sccm}\left(\mathrm{O}_{2}\right)$. For the samples studied in this paper, the oxidizing ions are not extracted $\left(\mathrm{V}^{+}=\mathrm{V}^{-}=0 \mathrm{~V}\right)$, reaching the sample with the thermal energy of $\sim 20 \mathrm{eV}$. Both for the deposition and oxidation, the table rotates at $15 \mathrm{rpm}$, and the table was tilted from $25^{\circ}$ to $80^{\circ}$ relatively to the loading direction.

The standard tunnel junctions described in this paper have the structure (thickness in angstroms): $\mathrm{Ta} 90 / \mathrm{Ni}_{80} \mathrm{Fe}_{20} 70 / \mathrm{Co}_{82} \mathrm{Fe}_{18}$ $30 / \mathrm{Al} 9+10$-s oxidation/ $\mathrm{Co}_{82} \mathrm{Fe}_{18} 40 / \mathrm{Mn}_{74} \mathrm{Ir}_{26} 250 / \mathrm{Ta} 30$, and were patterned by photolithography down to $2 \times 1 \mu \mathrm{m}^{2}$, using a self-aligned process.

\section{OXIDE LAYER CHARACTERIZATION}

\section{A. CoFe-Oxide Nanolayers}

Samples with the structure $\mathrm{Si} / \mathrm{Al}_{2} \mathrm{O}_{3} 600 \AA / \mathrm{Ta} 50 / \mathrm{CoFe}$ 100/oxidation/CoFe 100/Ta 50 (thickness in $\AA$ ) were analyzed 

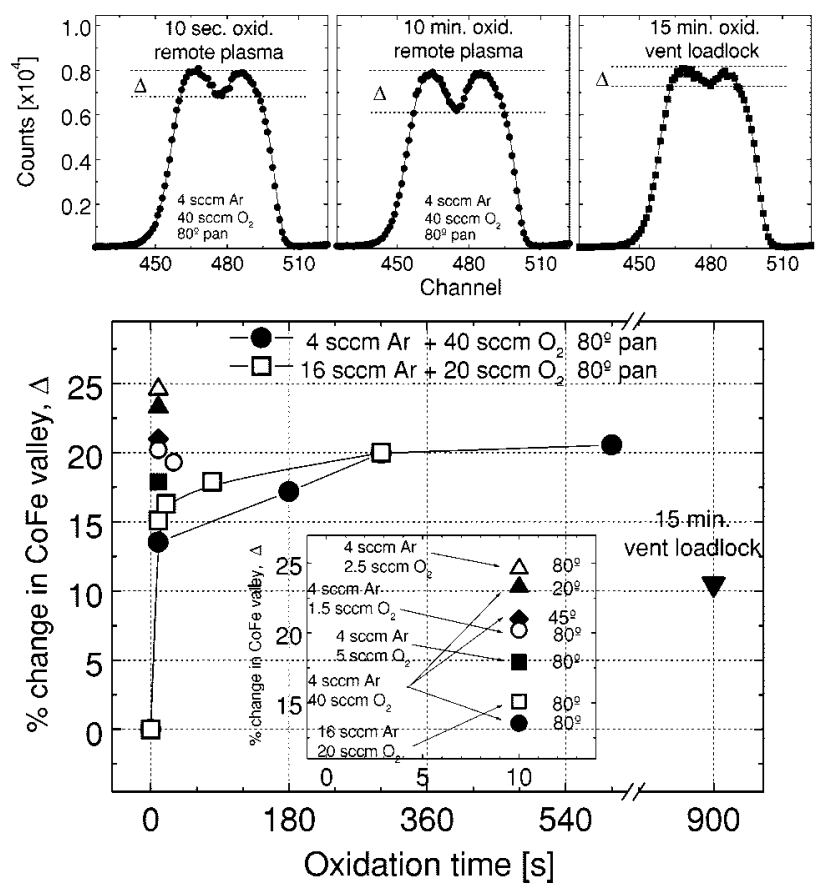

(b)

Fig. 2. (a) RBS spectra of the structure. Ta $50 \AA / \mathrm{CoFe} 100 \AA /$ oxidation/CoFe $100 \AA /$ Ta $50 \AA$. (b) Oxidation efficiency as a function of the oxidation time. The inset shows a detail for 10 -s oxidation.

using the Rutherford Backscattering Spectroscopy (RBS) technique, without any prior thermal treatment. For reference, one sample was prepared by natural oxidation: the bottom CoFe film was exposed to atmosphere for $15 \mathrm{~min}$ [4] in a class 100 clean room environment (30\% controlled humidity, $\left.20{ }^{\circ} \mathrm{C}\right)$. The RBS experiments were carried out with a $1.6-\mathrm{MeV}$ $\mathrm{He}^{+}$beam (collimated $0.2 \times 0.6 \mathrm{~mm}^{2}$ beam) in grazing angle incidence ( $77^{\circ}$ tilt), and the backscattering angle was $160^{\circ}$ in Cornell geometry. Information on the relative thickness of the oxide layers can be obtained directly from the experimental RBS spectra: without any oxidation, the RBS spectrum shows a single broad peak at the $\mathrm{Co}$ and $\mathrm{Fe}$ region (the width of the peak corresponding to the thickness of the two CoFe layers). When the bottom CoFe layer is oxidized (Fig. 2), the Co and $\mathrm{Fe}$ elements distribution in depth will change from a bulk layer to a mixed $\mathrm{Co}, \mathrm{Fe}$, and $\mathrm{O}$ layer, where the $\mathrm{CoFe}$ amount at a certain depth is reduced due to the presence of oxygen atoms. Consequently, the RBS spectra shows a valley between both $\mathrm{CoFe}$ layers, whose depth is proportional to the oxygen incorporated at the $\mathrm{CoFeO}$ layer. In Fig. 2(b), the percentage change $\Delta$ in the CoFe valley is used to compare samples with different oxidation conditions. The inset shows results on samples oxidized for $10 \mathrm{~s}$ under different oxidation conditions. This time was chosen because it is the required for complete oxidation of the 9- $\AA$-thick $\mathrm{Al}$ layer in the tunnel junction structures. Fig. 3 summarizes the oxidation $(\Delta)$ dependence on pan angle, oxidation pressure, and $\mathrm{O}_{2}$-Ar content during the oxidation. Lower pan angles $\left(20^{\circ}\right.$ and $\left.45^{\circ}\right)$ were chosen to avoid the substrate to be directly exposed to the assist source. The results actually indicate larger oxygen incorporation, when compared with an $80^{\circ}$ pan angle. This probably occurs because lower pan angles are associated with larger film exposed areas.

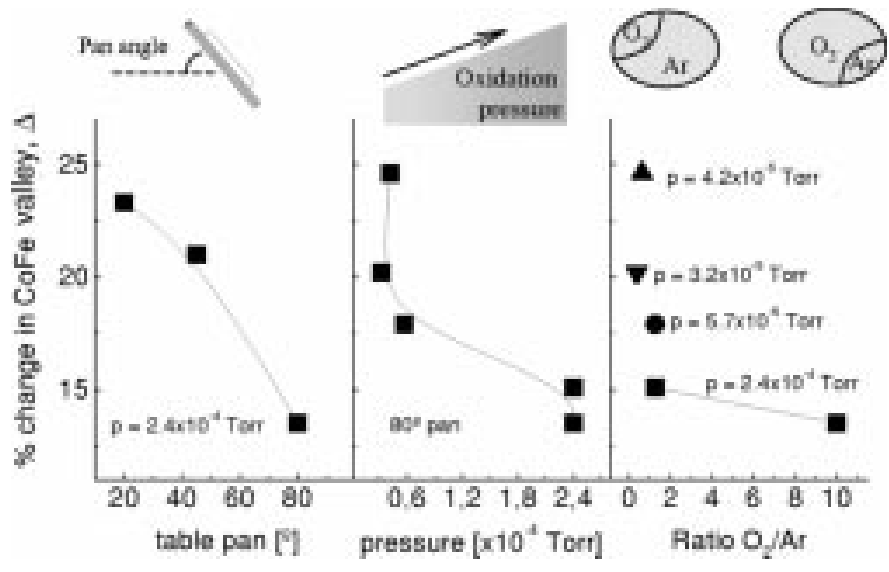

Fig. 3. Dependence of the CoFe valley depth on the oxidation conditions for 10-s oxidation

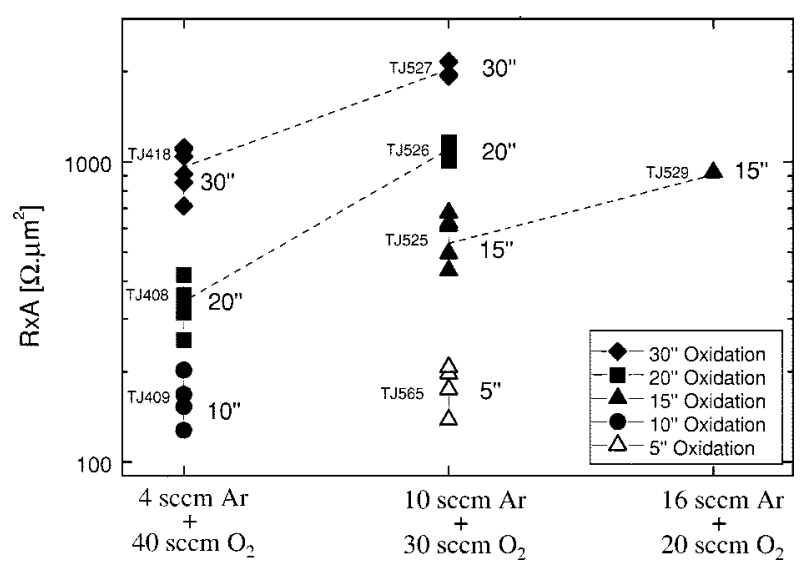

Fig. 4. Resistance-area products measured for junction devices fabricated with 9- $\AA$-thick $\mathrm{Al}$ layers. The oxidation was done with different $\mathrm{Ar}-\mathrm{O}_{2}$ ratios, while maintaining the same pressure $\left(2.4 \times 10^{-4}\right.$ torr $)$ and pan angle $\left(80^{\circ}\right)$.

It is also found that $\Delta$ decreases with oxidation pressure (less oxygen arriving at the film surface due to the decreased mean-free path). Keeping the same oxidation pressure, the oxidation efficiency decreases when decreasing the Ar content in the $\mathrm{Ar}-\mathrm{O}_{2}$ mixture. This result requires further investigation.

With the RBS technique, the absolute thickness values could only be obtained if the CoFe-oxide density is known. Accurate values were obtained by $\mathrm{X}$-rays, and the $\mathrm{CoFe}$-oxide thickness was calibrated as $30 \AA(\Delta=20 \%)$ and $15 \AA(\Delta=10 \%)$ [4]. The nature of the oxide cannot be identified, but X-ray photon spectroscopy (XPS) indicated clearly the formation of Fe-oxide phases and not Co-oxide.

\section{B. Al-Oxide Nanolayers in Tunnel Junctions}

Although the oxidation rate is different for $\mathrm{Co}, \mathrm{Fe}$, and $\mathrm{Al}$ films, the information obtained from the experiment was used when optimizing the oxidation of 9- $\AA$-thick Al layers in tunnel junction structures. Fig. 4 shows results from junctions fabricated with 10 -s oxidation at $80^{\circ}$ pan and $2.4 \times 10^{-4}$ torr, using different $\mathrm{Ar}-\mathrm{O}_{2}$ ratios. TMR values of $20 \%-26 \%$ are obtained for the as-deposited junctions. The increase of the junction resistance with the reduction of the $\mathrm{O}_{2}$ amount in the plasma is consistent with the increase of the CoFe valley depth plotted in 


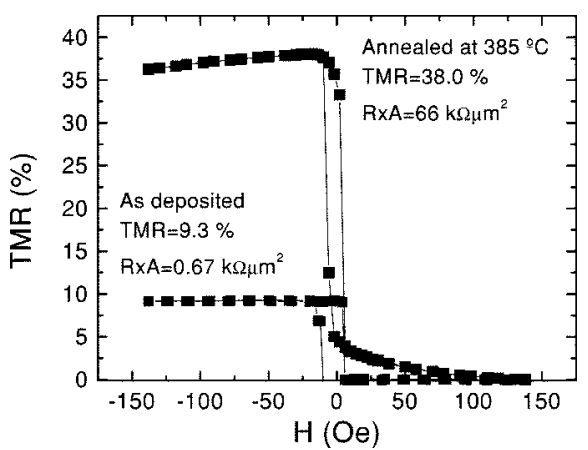

Fig. 5. Tunnel junction transfer curves as-deposited and after annealing at $385{ }^{\circ} \mathrm{C}$ (used with permission from [3]).

Fig. 3, indicating higher oxidation efficiency. After anneal (typically $1 \mathrm{~h}$ at $270^{\circ} \mathrm{C}$ ), oxygen migration from the partially oxidized interfacial $\mathrm{CoFe}$ into the barrier causes the TMR increase up to $\sim 45 \%$, with barrier heights $>2 \mathrm{eV}$, breakdown voltages of $\sim 1 \mathrm{~V}$, bias voltages $\mathrm{V}_{\text {half }}$ of $\sim 450 \mathrm{mV}$, and thermal stability up to $300{ }^{\circ} \mathrm{C}$ [5], which are good indicators of the high quality of the $\mathrm{Al}_{2} \mathrm{O}_{3}$ films. For thinner barriers, the bottom electrode roughness and the coverage of the $\mathrm{CoFe}$ layer by the ultrathin Al layer will determine the extension of the oxidation (partial oxidation of the bottom electrode will reduce the TMR), and in these cases, natural oxidation arises as a "softer" method for barrier fabrication [6].

\section{Fe-Oxide Nanolayers in Tunnel Junctions}

So as to increase the TMR values or improve the thermal stability, junctions have been fabricated with an Fe-oxide layer inserted between the barrier and the pinned top CoFe layer [3]. The goal is the formation of a ferromagnetic, half-metallic $\mathrm{Fe}_{3} \mathrm{O}_{4}$ electrode, with $100 \%$ polarization. However, the formation of this phase requires high-temperature growth, and the oxidation at room temperature of an Fe layer produces paramagnetic or antiferromagnetic phases instead [3]. We found (Fig. 5) that standard top pinned junctions (described in Section II) with a $25-\AA$-thick Fe layer oxidized for $10 \mathrm{~s}$ by remote plasma (conditions are the same as for the optimized 9- $\AA$ Al-layer oxidation) inserted between the AlOx barrier and the top pinned CoFe layer show low TMR in the as-deposited state $(9 \%)$ increasing sharply to $\sim 40 \%$ after anneal at $380{ }^{\circ} \mathrm{C}$ [3].

TEM analysis (Fig. 6) shows that in the as-deposited state this oxidation method leads to $\sim 18 \AA$ of metallic Fe and $\sim 22 \AA$ of $\mathrm{FeOx}$, with full oxidation of the Al layer (confirmed by XPS). The barrier is oxygen-deficient and becomes more stoichiometric upon anneal [7]. Magnetic measurements indicate that this "pure" $\mathrm{Fe}$ layer is paramagnetic. After annealing at $385^{\circ} \mathrm{C}$, the Fe layer becomes completely oxidized, with a $65-\AA$-thick homogeneous $\mathrm{FeOx}$ layer formed: $\mathrm{Fe}_{3} \mathrm{O}_{4}$ or $\mathrm{Fe}_{2} \mathrm{O}_{3}$ according to the interreticular spacing values of 0.29 and $0.25 \mathrm{~nm}$. The magnetic nature of this Fe-oxide layer was investigated. Magnetization measurements of $(\mathrm{Fe} 20-\AA / 10$-s oxidation) multilayers showed an increase of the moment upon anneal, coming from the as-deposited nonmagnetic or paramagnetic $\mathrm{FeOx}$ decomposition into a ferromagnetic oxide, thus $\mathrm{Fe}_{3} \mathrm{O}_{4}$.

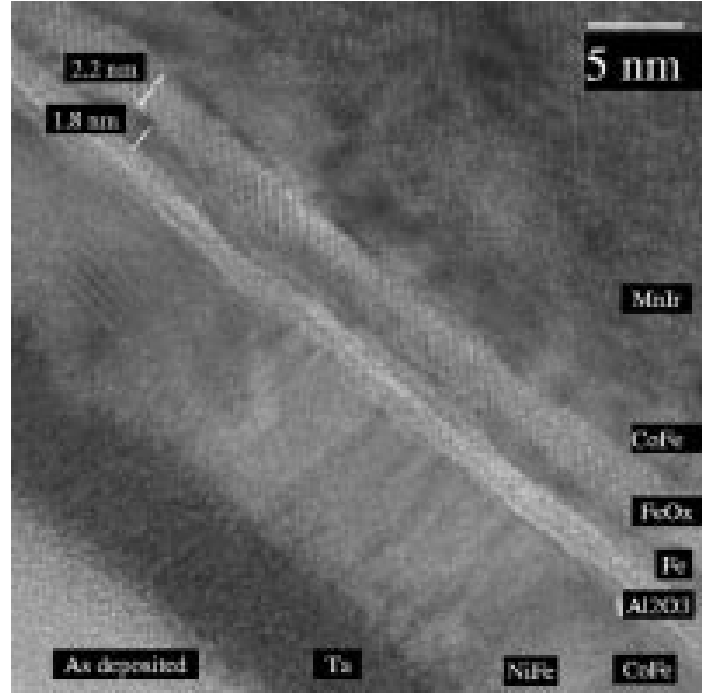

(a)

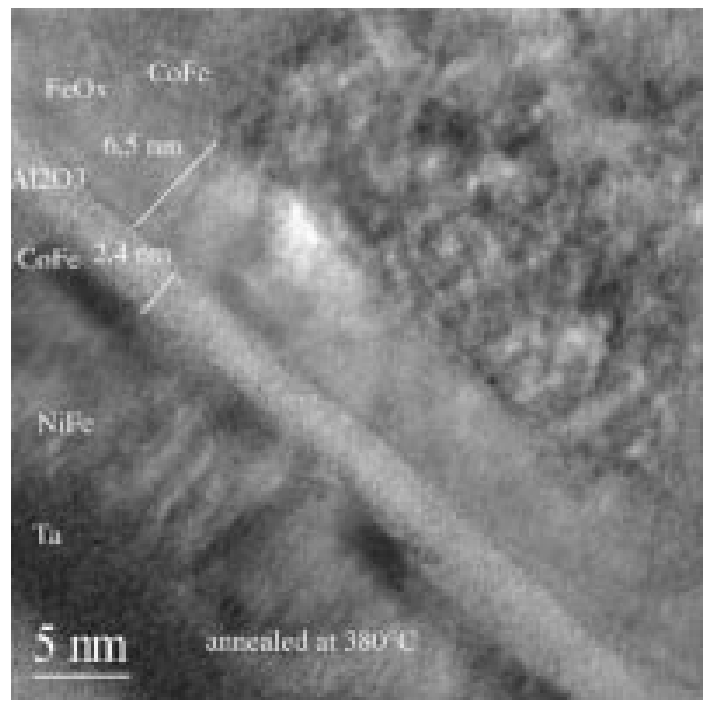

(b)

Fig. 6. Cross section high-resolution TEM pictures of the tunnel junction stack (a) as deposited and (b) after annealing at $380^{\circ} \mathrm{C}$.

\section{REFERENCES}

[1] J. Moodera, J. Nassar, and G. Mathon, "Spin tunneling in ferromagnetic junctions," Аnnu. Rev. Mater. Sci. 1999, vol. 29, pp. 381-432, 1999.

[2] S. Cardoso, V. Gehanno, R. Ferreira, and P. P. Freitas, "Ion beam deposition and oxidation of spin dependent tunnel junctions," IEEE Trans. Magn., vol. 35, pp. 2952-2954, Sept. 1999.

[3] Z. Zhang, S. Cardoso, P. P. Freitas, P. Wei, N. Barradas, and J. C. Soares, "Annealing effect of magnetic tunnel junctions with one $\mathrm{FeOx}$ layer inserted at the $\mathrm{Al}_{2} \mathrm{O}_{3} / \mathrm{CoFe}$ interface," Appl. Phys. Lett., vol. 78, pp. 2911-2913, May 2001.

[4] A. Veloso, P. P. Freitas, P. Wei, N. P. Barradas, J. C. Soares, B. Almeida, and J. B. Sousa, "Magnetoresistance enhancement in specular, bottompinned, MnIr spin valves with nano-oxide layers," Appl. Phys. Lett., vol. 77, pp. 1020-1022, Aug. 2000.

[5] P. P. Freitas, S. Cardoso, R. Sousa, W. Ku, and R. Ferreira, "Spin dependent tunnel junctions for memory and read head applications," IEEE Trans. Magn., vol. 36, pp. 2796-2801, Sept. 2000.

[6] S. Cardoso, Z. Zhang, and P. P. Freitas, "Electrode roughness and interfacial mixing effects on the tunnel junction thermal stability," J. Appl. Phys., vol. 89, pp. 6650-6652, 2001.

[7] A. E. T. Kuiper, M. F. Gillies, and V. Kottler et al., "Plasma oxidation of thin aluminum layers for magnetic spin-tunnel junctions," J. Appl. Phys., vol. 89, pp. 1965-1972, 2001. 